\title{
Mach-Type Soliton in the Novikov-Veselov Equation
}

Jen-Hsu CHANG

Department of Computer Science and Information Engineering, National Defense University, Tauyuan County 33551, Taiwan

E-mail: jhchang@ndu.edu.tw

Received September 18, 2014, in final form December 10, 2014; Published online December 18, 2014 http://dx.doi.org/10.3842/SIGMA.2014.111

\begin{abstract}
Using the reality condition of the solutions, one constructs the Mach-type soliton of the Novikov-Veselov equation by the minor-summation formula of the Pfaffian. We study the evolution of the Mach-type soliton and find that the amplitude of the Mach stem wave is less than two times of the one of the incident wave. It is shown that the length of the Mach stem wave is linear with time. One discusses the relations with $V$-shape initial value wave for different critical values of Miles parameter.
\end{abstract}

Key words: Pfaffian; Mach-type soliton; Mach stem wave; $V$-shape wave

2010 Mathematics Subject Classification: 35C08; 35A22

\section{Introduction}

Recently, the resonance theory of line solitons of KP-(II) equation (shallow water wave equation)

$$
\partial_{x}\left(-4 u_{t}+u_{x x x}+6 u u_{x}\right)+3 u_{y y}=0
$$

has attracted much attractions using the totally non-negative Grassmannian $[1,3,5,6,16$, $22,23]$, that is, those points of the real Grassmannian whose Plucker coordinates are all nonnegative. For the KP-(II) equation case, the $\tau$-function is described by the Wronskian form with respect to $x$. The Mach reflection problem describes the resonant interaction of solitary waves appearing in the reflection of an obliquely incident waves onto a vertical wall. John Miles discussed an oblique interaction of solitary waves and found a resonant interaction to describe the Mach reflection phenomena [26]. In this end, he predicts an extraordinary fourfold application of the wave at the wall. The Miles theory in terms of the KP equation and the Mach-type solution in KP observed experimentally are investigated in [16, 17, 18, 20, 31] (and references therein). The point is that irregular reflection can be described by the (3142)-type soliton and the stem in the middle part should be a Mach stem wave. Inspired by their works, one can consider the Novikov-Veselov equation similarly.

One considers the Novikov-Veselov (NV) equation $[4,11,15,28,30]$ with real solution $U$ :

$$
\begin{aligned}
& U_{t}=\operatorname{Re}\left[\partial_{z}^{3} U+3 \partial_{z}(Q U)-3 \epsilon \partial_{z} Q\right], \\
& \bar{\partial}_{z} Q=\partial_{z} U, \quad t \in R,
\end{aligned}
$$

where $\epsilon$ is a real constant. The NV equation (1.1) is one of the natural generation of the famous $\mathrm{KdV}$ equation and can have the Manakov's triad representation [24]

$$
L_{t}=[A, L]+B L
$$

where $L$ is the two-dimension Schrödinger operator

$$
L=\partial_{z} \bar{\partial}_{z}+U-\epsilon
$$


and

$$
A=\partial_{z}^{3}+Q \partial_{z}+\bar{\partial}_{z}^{3}+\bar{Q} \bar{\partial}_{z}, \quad B=Q_{z}+\bar{Q}_{\bar{z}}
$$

It is equivalent to the linear representation

$$
L \phi=0, \quad \partial_{t} \phi=A \phi .
$$

We remark that when $\epsilon \rightarrow \pm \infty$, the Veselov-Novikov equation reduces to the KP-I $(\epsilon \rightarrow-\infty)$ and KP-(II) $(\epsilon \rightarrow \infty)$ equation respectively [10]. To make a comparison with KP-(II) equation, we only consider $\epsilon>0$.

Let $\phi_{1}, \phi_{2}$ be any two independent solutions of (1.2). Then one can construct the extended Moutard transformation using the skew product $[2,13,25]$

$$
\begin{aligned}
W\left(\phi_{1}, \phi_{2}\right)= & \int\left(\phi_{1} \partial \phi_{2}-\phi_{2} \partial \phi_{1}\right) d z-\left(\phi_{1} \bar{\partial} \phi_{2}-\phi_{2} \bar{\partial} \phi_{1}\right) d \bar{z}+\left[\phi_{1} \partial^{3} \phi_{2}-\phi_{2} \partial^{3} \phi_{1}\right. \\
& +\phi_{2} \bar{\partial}^{3} \phi_{1}-\phi_{1} \bar{\partial}^{3} \phi_{2}+2\left(\partial^{2} \phi_{1} \partial \phi_{2}-\partial \phi_{1} \partial^{2} \phi_{2}\right)-2\left(\bar{\partial}^{2} \phi_{1} \bar{\partial} \phi_{2}-\bar{\partial} \phi_{1} \bar{\partial}^{2} \phi_{2}\right) \\
& \left.+3 Q\left(\phi_{1} \partial \phi_{2}-\phi_{2} \partial \phi_{1}\right)-3 \bar{Q}\left(\phi_{1} \bar{\partial} \phi_{2}-\phi_{2} \bar{\partial} \phi_{1}\right)\right] d t
\end{aligned}
$$

such that

$$
\hat{U}(t, z, \bar{z})=U(t, z, \bar{z})+2 \partial \bar{\partial} \ln W\left(\phi_{1}, \phi_{2}\right), \quad \hat{Q}(t, z, \bar{z})=Q(t, z, \bar{z})+2 \partial \partial \ln W\left(\phi_{1}, \phi_{2}\right),
$$

is also a solution of the NV equation (1.1).

For fixed potential $U_{0}(z, \bar{z}, t)$ and $Q_{0}(z, \bar{z}, t)$ of the NV equation (1.1), we can take any $2 N$ wave functions $\phi_{1}, \phi_{2}, \phi_{3}, \ldots, \phi_{2 N}$ (or their linear combinations) of (1.2). Then the $2 N$-step successive extended Moutard transformation can be expressed as the Pfaffian form [2, 27] (also see $[12,29])$

$$
U=U_{0}+2 \partial \bar{\partial}\left[\ln \operatorname{Pf}\left(\phi_{1}, \phi_{2}, \phi_{3}, \ldots, \phi_{2 N}\right)\right], \quad Q=Q_{0}+2 \partial \partial\left[\ln \operatorname{Pf}\left(\phi_{1}, \phi_{2}, \phi_{3}, \ldots, \phi_{2 N}\right)\right],
$$

where $\operatorname{Pf}\left(\phi_{1}, \phi_{2}, \phi_{3}, \ldots, \phi_{2 N}\right)$ is the Pfaffian defined by

$$
\operatorname{Pf}\left(\phi_{1}, \phi_{2}, \phi_{3}, \ldots, \phi_{2 N}\right)=\sum_{\sigma} \epsilon(\sigma) W_{\sigma_{1} \sigma_{2}} W_{\sigma_{3} \sigma_{4}} \cdots W_{\sigma_{2 N-1} \sigma_{2 N}}
$$

and $W_{\sigma_{i} \sigma_{j}}=W\left(\phi_{\sigma(i)}, \phi_{\sigma(j)}\right)$ is the extended Moutard transformation (1.3), $\sigma$ being some permutations.

To construct the $N$-solitons solutions, we take $V=U=0$ in (1.1) and then (1.2) becomes

$$
\partial \bar{\partial} \phi=\epsilon \phi, \quad \phi_{t}=\phi_{z z z}+\phi_{\bar{z} \bar{z} \bar{z}}
$$

where $\epsilon$ is non-zero real constant. The general solution of (1.4) can be expressed as

$$
\phi(z, \bar{z}, t)=\int_{\Gamma} e^{(i \lambda) z+(i \lambda)^{3} t+\frac{\epsilon}{i \lambda} \bar{z}+\frac{\epsilon^{3}}{(i \lambda)^{3}} t} \nu(\lambda) d \lambda,
$$

where $\nu(\lambda)$ is an arbitrary distribution and $\Gamma$ is an arbitrary path of integration such that the r.h.s. of (1.5) is well defined. One takes $\nu_{m}(\lambda)=\delta\left(\lambda-p_{m}\right)$, where $p_{m}$ is a complex number. Define

$$
\phi_{m}=\frac{\phi\left(p_{m}\right)}{\sqrt{3}}=\frac{1}{\sqrt{3}} e^{F\left(p_{m}\right)},
$$


where

$$
F(\lambda)=(i \lambda) z+(i \lambda)^{3} t+\frac{\epsilon}{i \lambda} \bar{z}+\frac{\epsilon^{3}}{(i \lambda)^{3}} t .
$$

Plugging $\left(\phi_{m}, \phi_{n}\right)$ into the extended Moutard transformation (1.3), we obtain

$$
W\left(\phi_{m}, \phi_{n}\right)=i \frac{p_{n}-p_{m}}{p_{n}+p_{m}} e^{F\left(p_{m}\right)+F\left(p_{n}\right)} .
$$

To study resonance, we introduce the real Grassmannian (or the $2 N \times M$ matrix) to construct $N$ solitons. To this end, one considers linear combination of $\phi_{n}$. Let

$$
\vec{\Psi}=\left(\phi_{1}, \phi_{2}, \phi_{3}, \ldots, \phi_{M}\right)^{T}
$$

and $H$ be an $2 N \times M(2 N \leq M)$ of real constant matrix (or Grassmannian). Suppose that

$$
H \vec{\Psi}=\vec{\Psi}^{*}=\left(\Psi_{1}^{*}, \Psi_{2}^{*}, \Psi_{3}^{*}, \ldots, \Psi_{2 N}^{*}\right)^{T},
$$

that is,

$$
\Psi_{n}^{*}=h_{n 1} \phi_{1}+h_{n 2} \phi_{2}+h_{n 3} \phi_{3}+\cdots+h_{n M} \phi_{M}, \quad 1 \leq n \leq 2 N .
$$

Then one has by the minor-summation formula $[14,19]$

$$
\tau_{N}=\operatorname{Pf}\left(\Psi_{1}^{*}, \Psi_{2}^{*}, \Psi_{3}^{*}, \ldots, \Psi_{2 N}^{*}\right)=\operatorname{Pf}\left(H W_{M} H^{T}\right)=\sum_{I \subset[M], \sharp I=2 N} \operatorname{Pf}\left(H_{I}^{I}\right) \operatorname{det}\left(H_{I}\right),
$$

where the $M \times M$ matrix $W_{M}$ is defined by the element (1.6) and $H_{I}^{I}$ denote the $2 N \times M$ submatrix of $H$ obtained by picking up the rows and columns indexed by the same index set $I$. By this formula, the resonance of real solitons of the Novikov-Veselov equation can be investigated just like the resonance theory of $\operatorname{KP}$-(II) equation [16, 21, 23]. Finally, the $N$-solitons solutions are defined by $[7,9]$

$$
U(z, \bar{z}, t)=2 \partial \bar{\partial} \ln \tau_{N}(z, \bar{z}, t), \quad V(z, \bar{z}, t)=2 \partial \partial \ln \tau_{N}(z, \bar{z}, t) .
$$

To obtain the real potential $U$, the following reality conditions [9] for resonance have to be considered

$$
\left|p_{k}\right|^{2}=\left|q_{k}\right|^{2}=\epsilon>0, \quad k=1,2,3, \ldots, m,
$$

given $m$ pairs of complex numbers $\left(p_{1}, q_{1}\right),\left(p_{2}, q_{2}\right), \ldots,\left(p_{m}, q_{m}\right)$.

Letting $p_{m}=\sqrt{\epsilon} e^{i \alpha_{m}}$ and removing $i$ factor from (1.6) afterwards, one has

$$
W\left(\phi_{m}, \phi_{n}\right)=-\tan \frac{\alpha_{n}-\alpha_{m}}{2} e^{\phi_{m n}},
$$

where

$$
\begin{aligned}
\phi_{m n}= & F\left(p_{m}\right)+F\left(p_{n}\right)=-2 \sqrt{\epsilon}\left[x\left(\sin \alpha_{m}+\sin \alpha_{n}\right)+y\left(\cos \alpha_{m}+\cos \alpha_{n}\right)\right] \\
& +2 t \epsilon \sqrt{\epsilon}\left(\sin 3 \alpha_{m}+\sin 3 \alpha_{n}\right) .
\end{aligned}
$$

Therefore, given a $2 N \times M$ matrix $H$, the associated $\tau_{H}$-function can be written as by (1.7) [8]

$$
\tau_{H}=\sum_{I \subset[M], \sharp I=2 N} \Gamma_{I} \Lambda_{I}(x, y, t)
$$


where

$$
\Lambda_{I}(x, y, t)=\operatorname{Pf}\left(W_{2 N}\right)=(-1)^{N}\left(\prod_{i=2, i>j}^{2 N} \tan \frac{\alpha_{i}-\alpha_{j}}{2}\right) e^{\sum_{m=1}^{2 N} F\left(p_{m}\right)},
$$

$\Gamma_{I}$ being the $2 N \times 2 N$ minor for the columns with the index set $I=\left\{i_{1}, i_{2}, i_{3}, \ldots, i_{2 N}\right\}$. Also, to keep $\tau_{H}$ totally positive (or totally negative), we assume that the matrix $H$ belongs to the totally non-negative Grassmannian $[22,23]$ and the angle $\alpha_{n}$ satisfies the following condition:

$$
-\frac{\pi}{2} \leq \alpha_{1}<\alpha_{2}<\alpha_{3}<\cdots<\alpha_{M-1}<\alpha_{M} \leq \frac{\pi}{2} .
$$

For one-soliton solution, we have, $-\frac{\pi}{2} \leq \alpha_{i}<\alpha_{j}<\alpha_{k} \leq \frac{\pi}{2}$

$$
\begin{aligned}
\tau_{1} & =\tan \frac{\alpha_{i}-\alpha_{j}}{2} e^{\phi_{i j}}+a \tan \frac{\alpha_{i}-\alpha_{k}}{2} e^{\phi_{i k}} \\
& =a e^{\phi_{i k}} \tan \frac{\alpha_{i}-\alpha_{k}}{2}\left[1+\frac{1}{a} \frac{\tan \frac{\alpha_{i}-\alpha_{j}}{2}}{\tan \frac{\alpha_{i}-\alpha_{k}}{2}} e^{\left.F\left(p_{j}\right)-F\left(p_{k}\right)\right]}\right. \\
& =a e^{\phi_{i k}} \tan \frac{\alpha_{i}-\alpha_{k}}{2}\left[1+e^{F\left(p_{j}\right)-F\left(p_{k}\right)+\theta_{j k}}\right],
\end{aligned}
$$

where $a$ is a constant and the phase shift

$$
\theta_{j k}=\ln \frac{1}{a} \frac{\tan \frac{\alpha_{i}-\alpha_{j}}{2}}{\tan \frac{\alpha_{i}-\alpha_{k}}{2}}=\ln \frac{\tan \frac{\alpha_{i}-\alpha_{j}}{2}}{\tan \frac{\alpha_{i}-\alpha_{k}}{2}}-\ln a .
$$

Hence the real one-soliton solution is [8]

$$
\begin{aligned}
U & =2 \partial_{z} \partial_{\bar{z}} \ln a e^{\phi_{i k}} \tan \frac{\alpha_{i}-\alpha_{k}}{2}\left[1+e^{F\left(p_{j}\right)-F\left(p_{k}\right)+\theta_{j k}}\right]=2 \partial_{z} \partial_{\bar{z}}\left[1+e^{F\left(p_{j}\right)-F\left(p_{k}\right)+\theta_{j k}}\right] \\
& =\frac{1}{2}\left|p_{k}-p_{j}\right|^{2} \operatorname{sech}^{2}\left[\frac{F\left(p_{j}\right)-F\left(p_{k}\right)+\theta_{j k}}{2}\right] \\
& =2 \epsilon \sin ^{2}\left(\frac{\alpha_{k}-\alpha_{j}}{2}\right) \operatorname{sech}^{2}\left[\frac{F\left(p_{j}\right)-F\left(p_{k}\right)+\theta_{j k}}{2}\right] \\
& =A_{[j, k]} \operatorname{sech}^{2} \frac{1}{2}\left(\overrightarrow{\mathbf{K}}_{[j, k]} \cdot \vec{x}-\Omega_{[j, k]} t+\theta_{j k}\right) .
\end{aligned}
$$

From (1.9) the amplitude $A_{[j, k]}$, the wave vector $\overrightarrow{\mathbf{K}}_{[j, k]}$ and the frequency $\boldsymbol{\Omega}_{[j, k]}$ are defined by

$$
\begin{aligned}
& A_{[j, k]}=2 \epsilon \sin ^{2}\left(\frac{\alpha_{k}-\alpha_{j}}{2}\right), \\
& \overrightarrow{\mathbf{K}}_{[j, k]}=2 \sqrt{\epsilon}\left(-\sin \alpha_{j}+\sin \alpha_{k},-\cos \alpha_{j}+\cos \alpha_{k}\right), \\
& \boldsymbol{\Omega}_{[j, k]}=2 \epsilon \sqrt{\epsilon}\left[-\sin 3 \alpha_{j}+\sin 3 \alpha_{k}\right],
\end{aligned}
$$

The direction of the wave vector $\overrightarrow{\mathbf{K}}_{[j, k]}=\left(K_{[j, k]}^{x}, K_{[j, k]}^{y}\right)$ is measured in the clockwise sense from the $y$-axis and it is given by

$$
\frac{K_{[j, k]}^{y}}{K_{[j, k]}^{x}}=\frac{-\cos \alpha_{j}+\cos \alpha_{k}}{-\sin \alpha_{j}+\sin \alpha_{k}}=-\tan \frac{\alpha_{j}+\alpha_{k}}{2},
$$

that is, $\frac{\alpha_{j}+\alpha_{k}}{2}$ gives the angle between the line soliton and the $y$-axis in the clockwise sense. In addition, the soliton velocity $\mathbf{V}_{[\mathbf{j}, \mathbf{k}]}$ is [8]

$$
\mathbf{V}_{[\mathbf{j}, \mathbf{k}]}=\frac{\epsilon}{4} \frac{\sin 3 \alpha_{k}-\sin 3 \alpha_{j}}{\sin ^{2} \frac{\alpha_{j}-\alpha_{k}}{2}}\left(\sin \alpha_{k}-\sin \alpha_{j}, \cos \alpha_{k}-\cos \alpha_{j}\right) .
$$


The paper is organized as follows. In Section 2, one investigates Mach-type or (3142)-type soliton for the Novikov-Veselov equation. One shows the evolution of the Mach-type soliton and obtains the relation of the amplitude of the Mach stem wave $([1,4]$-soliton) with the one of the incident wave ([1,3]-soliton). Furthermore, the length of the Mach stem wave is linear with time. In Section 3, we discuss the relations with $V$-shape initial value wave for different critical value of Miles parameter $\kappa$. It is shown that the amplitude of the Mach stem wave is less than two times of the one of the incident wave. In Section 4, we conclude the paper with several remarks.

\section{Mach type soliton}

In this section, we investigate the Mach-type or (3142)-type soliton. The corresponding totally non-negative Grassmannian is the the matrix [16]

$$
H_{M}=\left[\begin{array}{cccc}
1 & a & 0 & -c \\
0 & 0 & 1 & b
\end{array}\right]
$$

where $a, b, c$ are positive numbers. When $c=0$, one has the $O$-type soliton for the NovikovVeselov equation. For $V$-shape initial value wave, one can introduce parameter $\kappa$ to determine the evolution into Mach-type or $O$-type soliton (see next section). We remark that the $Y$ shape, $O$-type, and $P$-type solitons for the Novikov-Veselov equation are investigated in [8]. Now,

$$
H_{M} \frac{1}{\sqrt{3}}\left[\phi\left(p_{1}\right), \phi\left(p_{2}\right), \phi\left(p_{3}\right), \phi\left(p_{4}\right)\right]^{T}=\frac{1}{\sqrt{3}}\left[\begin{array}{c}
\phi\left(p_{1}\right)+a \phi\left(p_{2}\right) \\
\phi\left(p_{3}\right)+b \phi\left(p_{4}\right)
\end{array}\right]=\left[\begin{array}{c}
\Psi_{1}^{*} \\
\Psi_{2}^{*}
\end{array}\right]
$$

A direct calculation yields by (1.7), (1.8) and (1.9)

$$
\begin{aligned}
\tau_{M}= & W\left(\Psi_{1}^{*}, \Psi_{2}^{*}\right)=W\left(\phi_{1}, \phi_{3}\right)+b W\left(\phi_{1}, \phi_{4}\right)+a W\left(\phi_{2}, \phi_{3}\right)+a b W\left(\phi_{2}, \phi_{4}\right)+c W\left(\phi_{3}, \phi_{4}\right) \\
= & \tan \frac{\alpha_{1}-\alpha_{3}}{2} e^{-2 \sqrt{\epsilon}\left[x\left(\sin \alpha_{1}+\sin \alpha_{3}\right)+y\left(\cos \alpha_{1}+\cos \alpha_{3}\right)\right]+2 t \epsilon \sqrt{\epsilon}\left(\sin 3 \alpha_{1}+\sin 3 \alpha_{3}\right)} \\
& +b \tan \frac{\alpha_{1}-\alpha_{4}}{2} e^{-2 \sqrt{\epsilon}\left[x\left(\sin \alpha_{1}+\sin \alpha_{4}\right)+y\left(\cos \alpha_{1}+\cos \alpha_{4}\right)\right]+2 t \epsilon \sqrt{\epsilon}\left(\sin 3 \alpha_{1}+\sin 3 \alpha_{4}\right)} \\
& +a \tan \frac{\alpha_{2}-\alpha_{3}}{2} e^{-2 \sqrt{\epsilon}\left[x\left(\sin \alpha_{2}+\sin \alpha_{3}\right)+y\left(\cos \alpha_{2}+\cos \alpha_{3}\right)\right]+2 t \epsilon \sqrt{\epsilon}\left(\sin 3 \alpha_{2}+\sin 3 \alpha_{3}\right)} \\
& +a b \tan \frac{\alpha_{2}-\alpha_{4}}{2} e^{-2 \sqrt{\epsilon}\left[x\left(\sin \alpha_{2}+\sin \alpha_{4}\right)+y\left(\cos \alpha_{2}+\cos \alpha_{4}\right)\right]+2 t \epsilon \sqrt{\epsilon}\left(\sin 3 \alpha_{2}+\sin 3 \alpha_{4}\right)} \\
& +c \tan \frac{\alpha_{3}-\alpha_{4}}{2} e^{-2 \sqrt{\epsilon}\left[x\left(\sin \alpha_{3}+\sin \alpha_{4}\right)+y\left(\cos \alpha_{3}+\cos \alpha_{4}\right)\right]+2 t \epsilon \sqrt{\epsilon}\left(\sin 3 \alpha_{3}+\sin 3 \alpha_{4}\right)},
\end{aligned}
$$

where

$$
-\frac{\pi}{2} \leq \alpha_{1}<\alpha_{2}<\alpha_{3}<\alpha_{4} \leq \frac{\pi}{2} .
$$

To investigate the asymptotic behavior for $|y| \rightarrow \infty$, we use the notation [16], considering the line $x=-c y$,

$$
\eta_{m}(c)=-c \sin \alpha_{m}+\cos \alpha_{m} .
$$

When $\eta_{m}(c)=\eta_{n}(c)$, one gets

$$
c=\frac{\cos \alpha_{m}-\cos \alpha_{n}}{\sin \alpha_{m}-\sin \alpha_{n}}=-\tan \frac{\alpha_{m}+\alpha_{n}}{2} .
$$


Since

$$
\begin{aligned}
{\left.\left[\eta_{m}(c)-\eta_{i}(c)\right]\right|_{c=-\tan \frac{\alpha_{i}+\alpha_{j}}{2}} } & =\cos \alpha_{m}-\cos \alpha_{i}+\tan \frac{\alpha_{i}+\alpha_{j}}{2}\left(\sin \alpha_{m}-\sin \alpha_{i}\right) \\
& =\left(\sin \alpha_{m}-\sin \alpha_{i}\right)\left[\tan \frac{\alpha_{i}+\alpha_{j}}{2}-\tan \frac{\alpha_{i}+\alpha_{m}}{2}\right],
\end{aligned}
$$

we have the following order relations among the other $\eta_{m}(c)^{\prime} s$ along $c=-\tan \frac{\alpha_{i}+\alpha_{j}}{2}$

$$
\begin{cases}\eta_{i}=\eta_{j}<\eta_{m} & \text { if } \quad i<m<j \\ \eta_{i}=\eta_{j}>\eta_{m} & \text { if } \quad m<i \quad \text { or } \quad m>j .\end{cases}
$$

Then by a similar argument in [16], one knows that by (1.10):

(a) For $y \gg 0$, there are two unbounded line solitons, whose types from left to right are

$$
[1,3], \quad[3,4] .
$$

(b) For $y \ll 0$, there are two unbounded line solitons, whose types from left to right are

$$
[4,2], \quad[2,1] .
$$

It can be verified by the Maple software.

Now, we can discuss the relations between the parameters $a, b, c$ and phase shifts of these line solitons. Let us first consider the line solitons in $x \gg 0$. There are two solitons which are [3,4]soliton and $[2,1]$-soliton. The $[3,4]$-soliton is obtained by the balance between the exponential terms $W\left(\phi_{1}, \phi_{3}\right)$ and $b W\left(\phi_{1}, \phi_{4}\right)$, and the [2,1]-soliton is obtained by the balance between the exponential terms $W\left(\phi_{1}, \phi_{3}\right)$ and $a W\left(\phi_{2}, \phi_{3}\right)$. Therefore, the phase shifts of [3,4]-soliton and $[2,1]$-soliton for $x \gg 0$ are given by

$$
\theta_{[3,4]}^{+}=\ln \frac{\tan \frac{\alpha_{3}-\alpha_{1}}{2}}{\tan \frac{\alpha_{4}-\alpha_{1}}{2}}-\ln b, \quad \theta_{[2,1]}^{+}=\ln \frac{\tan \frac{\alpha_{3}-\alpha_{1}}{2}}{\tan \frac{\alpha_{3}-\alpha_{2}}{2}}-\ln a .
$$

For the line solitons in $x \ll 0$, there are two solitons, which are [1,3]-soliton and [4,2]-soliton. The [1,3]-soliton is obtained by the balance between the exponential terms $c W\left(\phi_{3}, \phi_{4}\right)$ and $b W\left(\phi_{1}, \phi_{4}\right)$, and the $[4,2]$-soliton is obtained by the balance between the exponential terms $c W\left(\phi_{3}, \phi_{4}\right)$ and $a W\left(\phi_{2}, \phi_{3}\right)$. Therefore, the phase shifts of [1,3]-soliton and [4,2]-soliton for $x \ll 0$ are given by

$$
\theta_{[1,3]}^{-}=\ln \frac{\tan \frac{\alpha_{4}-\alpha_{1}}{2}}{\tan \frac{\alpha_{4}-\alpha_{3}}{2}}+\ln \frac{b}{c}, \quad \theta_{[4,2]}^{-}=\ln \frac{\tan \frac{\alpha_{3}-\alpha_{2}}{2}}{\tan \frac{\alpha_{4}-\alpha_{3}}{2}}+\ln \frac{a}{c} .
$$

So one can see that

$$
\theta_{[1,3]}^{-}+\theta_{[3,4]}^{+}=\theta_{[4,2]}^{-}+\theta_{[2,1]}^{+}=\text {total phase shift }=\ln \frac{\tan \frac{\alpha_{3}-\alpha_{1}}{2}}{\tan \frac{\alpha_{4}-\alpha_{3}}{2}}-\ln c .
$$

We define the parameter $s$ (representing the total phase shift) by $s=e^{-\theta_{[4,2]}^{-}-\theta_{[2,1]}^{+}}$, which leads to

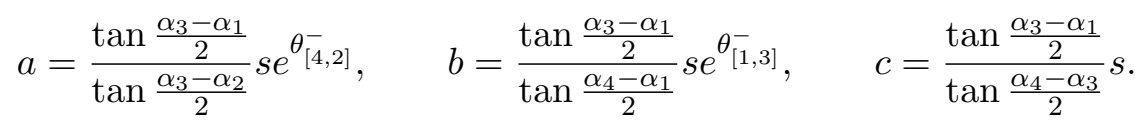

Hence we know that the three parameters $a, b, c$ can be used to determine the locations of three asymptotic line solitons, that is, two in $x \ll 0$ and one in $x>0$. The $s$-parameter represents the 


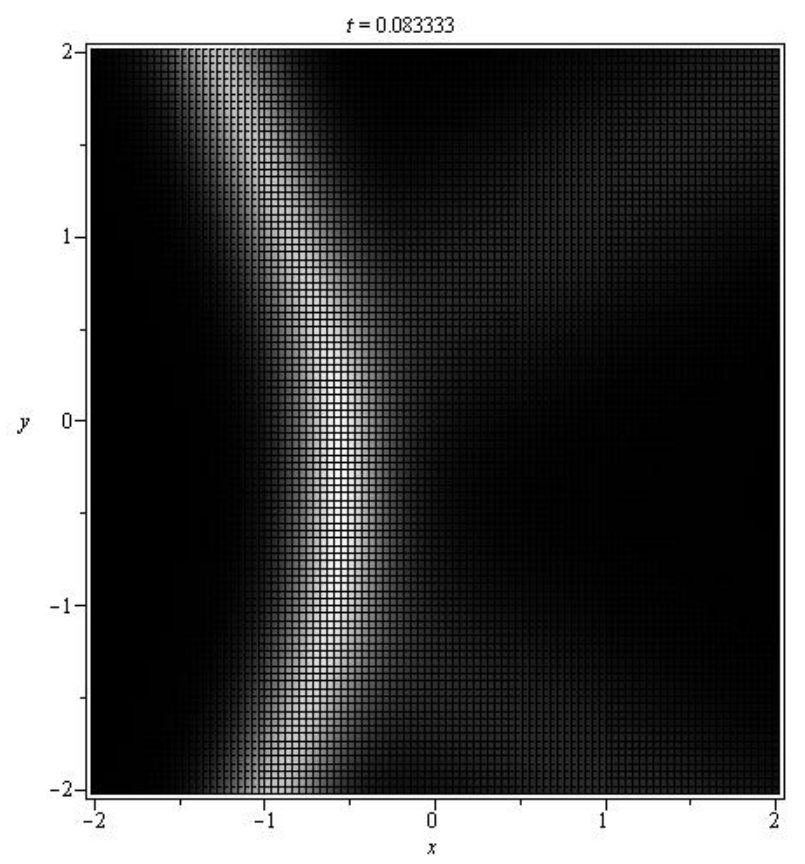

Figure 1. The middle portion, having maximum amplitude, is the $[1,4]$-soliton (stem wave). The $y$-axis is slightly enlarged to make the middle portion longer.

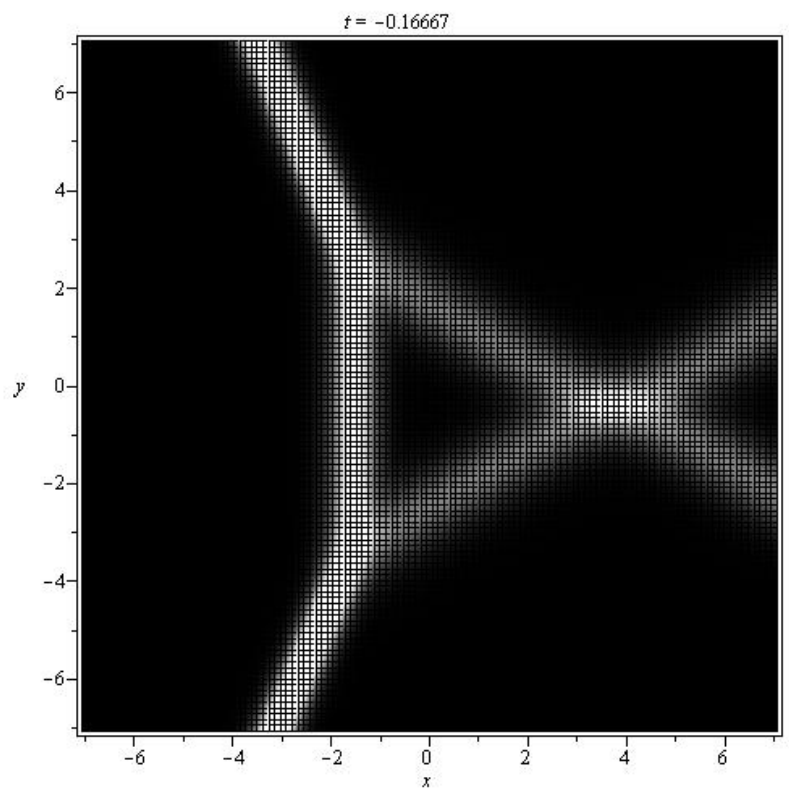

Figure 2. $\alpha_{1}=-\frac{23}{50} \pi, \alpha_{2}=-\frac{1}{5} \pi, \alpha_{3}=\frac{1}{5} \pi, \alpha_{4}=\frac{23}{50} \pi, a=b=c=1, \epsilon=5$.

relative locations of the intersection point of the [1,3]-soliton and [3,4]-soliton with the $x$-axis. Especially, when $s=1, \theta_{[4,2]}^{-}=0, \theta_{[1,3]}^{-}=0$, all of the four solitons will intersect at $(0,0)$ when $t=0$. One remarks that the bounded line soliton $[1,4]$ (Mach stem wave), obtained by the balance between the exponential terms $W\left(\phi_{1}, \phi_{3}\right)$ and $c W\left(\phi_{3}, \phi_{4}\right)$, has the maximal amplitude among all the solitons by (1.11) (Fig. 1) and the velocity is obtained by (1.12). Furthermore, when $t<0$, there is a bounded line [2,3]-soliton (Fig. 2, the left side of the triangle), obtained by the balance between the exponential terms $a b W\left(\phi_{2}, \phi_{4}\right)$ and $c W\left(\phi_{3}, \phi_{4}\right)$. 


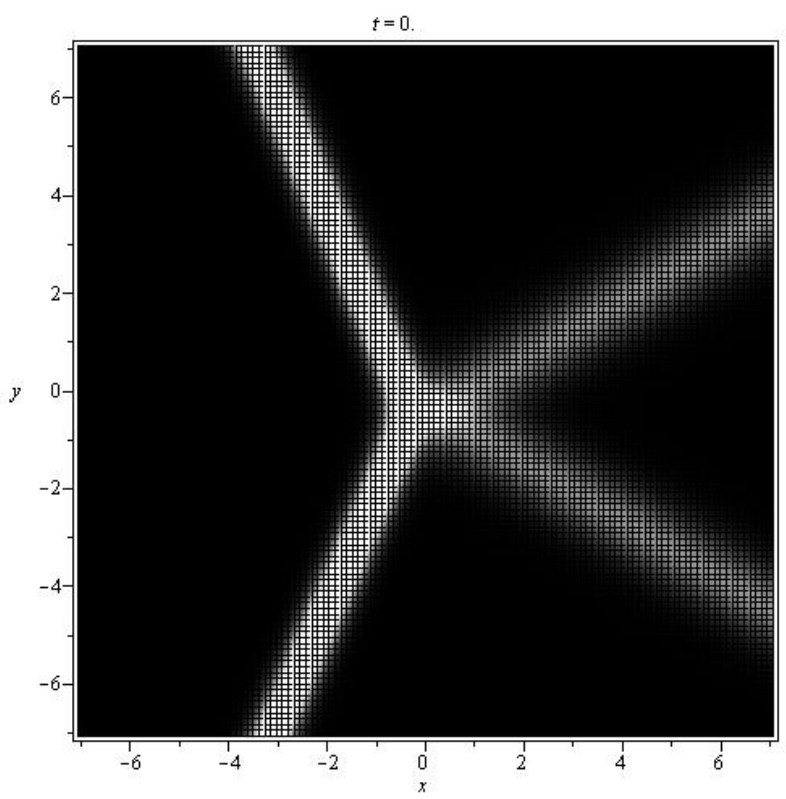

Figure 3. Initial wave.

Now, we consider the case $\alpha_{3}=-\alpha_{2} \geq 0, \alpha_{4}=-\alpha_{1} \geq 0$, and the amplitude

$$
A=A_{[1,3]}=A_{[4,2]} \leq 2 \epsilon
$$

is fixed. Then one can see that $[1,3]$-soliton and $[4,2]$-soliton is symmetric to the $x$-axis and similarly for $[3,4]$-soliton and $[2,1]$-soliton. By $(1.11)$, one knows

$$
\frac{\alpha_{3}+\alpha_{1}}{2} \leq \frac{\alpha_{3}-\alpha_{1}}{2}=\frac{\alpha_{3}+\alpha_{4}}{2}=\arcsin \sqrt{\frac{A}{2 \epsilon}} .
$$

Therefore the angle between the [1,3]-soliton and the $y$-axis (counter-clockwise) is less than the the angle between the [3,4]-soliton and the $y$-axis (clockwise). We see that given $A$ and $2 \epsilon$ there is a critical angle $\varphi_{C}=\arcsin \sqrt{\frac{A}{2 \epsilon}}$ for the angle between the $[1,3]$-soliton and the $y$-axis (counter-clockwise). Then one can introduce the following Miles-parameter $[5,8,26]$ to describe the interaction for the Mach-type solution, noticing $\frac{\alpha_{3}+\alpha_{1}}{2} \leq 0$,

$$
\kappa=\frac{\left|\tan \frac{\alpha_{3}+\alpha_{1}}{2}\right|}{\tan \frac{\alpha_{3}+\alpha_{4}}{2}}=\frac{\left|\tan \frac{\alpha_{3}+\alpha_{1}}{2}\right|}{\tan \varphi_{C}}=\frac{\left|\tan \frac{\alpha_{3}+\alpha_{1}}{2}\right|}{\sqrt{\frac{A}{2 \epsilon-A}}} \leq 1 .
$$

From (1.11), we have thus using $\kappa$

$$
\begin{gathered}
A=A_{[1,3]}=A_{[4,2]}=\frac{2 \epsilon\left(\tan \varphi_{C}\right)^{2}}{1+\left(\tan \varphi_{C}\right)^{2}}, \quad A_{[3,4]}=A_{[2,1]}=\frac{2 \epsilon\left(\tan \varphi_{C}\right)^{2}}{\frac{1}{\kappa^{2}}+\left(\tan \varphi_{C}\right)^{2}} \leq A, \\
A_{[1,4]}=2 \epsilon \sin ^{2} \frac{\alpha_{4}-\alpha_{1}}{2}=2 \epsilon\left[\sin \left(\frac{\alpha_{4}-\alpha_{3}}{2}+\frac{\alpha_{3}-\alpha_{1}}{2}\right)\right]^{2} \\
=\frac{2 \epsilon\left(\tan \varphi_{C}\right)^{2}(\kappa+1)^{2}}{\left[1+\left(\tan \varphi_{C}\right)^{2}\right]\left[1+\kappa^{2}\left(\tan \varphi_{C}\right)^{2}\right]}=A \frac{(\kappa+1)^{2}}{\left[1+\kappa^{2}\left(\tan \varphi_{C}\right)^{2}\right]}<4 A .
\end{gathered}
$$

Remark. To make a comparison with KP-(II), we see that

$$
2 \epsilon-A=2 \epsilon\left(1-\sin ^{2} \frac{\alpha_{3}-\alpha_{1}}{2}\right)=2 \epsilon \cos ^{2} \frac{\alpha_{3}-\alpha_{1}}{2} .
$$




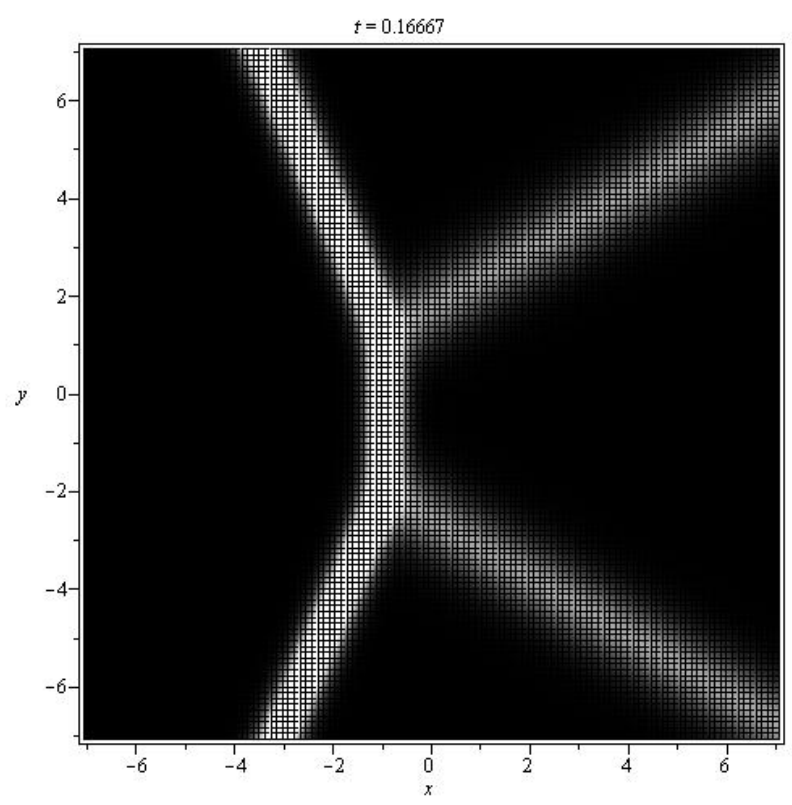

Figure 4. The stem wave moves to the left.

When $\epsilon \rightarrow \infty, \alpha_{1} \rightarrow-\frac{\pi}{2}$ and $\alpha_{3} \rightarrow \frac{\pi}{2}$ such that

$$
\epsilon \cos ^{2} \frac{\alpha_{3}-\alpha_{1}}{2}=\frac{1}{4}
$$

Then

$$
\kappa \rightarrow \frac{\left|\tan \frac{\alpha_{3}+\alpha_{1}}{2}\right|}{\sqrt{2 A}}
$$

which is the Miles parameter of KP-(II) to describe the interactions of water wave solitons [16, $17,18]$.

Since the [1,4]-soliton (Mach stem wave) is increasing its length with time but its end points will lie in a line (see Figs. 4 and 5), we can obtain them as follows.

We choose $s=1, \theta_{[4,2]}^{-}=0, \theta_{[1,3]}^{-}=0$ such that [1,3]-soliton and [1,4]-soliton will intersect at $(0,0)$ when $t=0$ (see Fig. 3). From (1.10)), the ridges of [1,3]-soliton and [1,4]-soliton are given by $F\left(p_{1}\right)-F\left(p_{3}\right)=0, F\left(p_{1}\right)-F\left(p_{4}\right)=0$, which lead to

$$
\begin{aligned}
& x\left(-\sin \alpha_{1}+\sin \alpha_{3}\right)+y\left(-\cos \alpha_{1}+\cos \alpha_{3}\right)+t \epsilon\left(\sin 3 \alpha_{1}-\sin 3 \alpha_{3}\right)=0, \\
& x\left(-\sin \alpha_{1}+\sin \alpha_{4}\right)+y\left(-\cos \alpha_{1}+\cos \alpha_{4}\right)+t \epsilon\left(\sin 3 \alpha_{1}-\sin 3 \alpha_{4}\right)=0 .
\end{aligned}
$$

Noticing that $\alpha_{3}=-\alpha_{2} \geq 0, \alpha_{4}=-\alpha_{1} \geq 0$, one gets

$$
\begin{aligned}
x & =\frac{t \epsilon \sin 3 \alpha_{4}}{\sin \alpha_{4}}=t \epsilon\left(4 \cos ^{2} \alpha_{4}-1\right), \\
y & =\frac{x\left(\sin \alpha_{1}-\sin \alpha_{3}\right)+t \epsilon\left(-\sin 3 \alpha_{1}+\sin 3 \alpha_{3}\right)}{-\cos \alpha_{1}+\cos \alpha_{3}} \\
& =t \epsilon \frac{\left(4 \cos ^{2} \alpha_{4}-1\right)\left(\sin \alpha_{1}-\sin \alpha_{3}\right)+\left(-\sin 3 \alpha_{1}+\sin 3 \alpha_{3}\right)}{-\cos \alpha_{1}+\cos \alpha_{3}} \\
& =4 t \epsilon \frac{\sin \alpha_{3}\left(\sin \alpha_{3}+\sin \alpha_{4}\right)\left(\sin \alpha_{4}-\sin \alpha_{3}\right)}{\cos \alpha_{3}-\cos \alpha_{4}}=4 t \epsilon \sin \alpha_{3}\left(\sin \alpha_{3}+\sin \alpha_{4}\right) \cot \frac{\alpha_{3}+\alpha_{4}}{2} .
\end{aligned}
$$




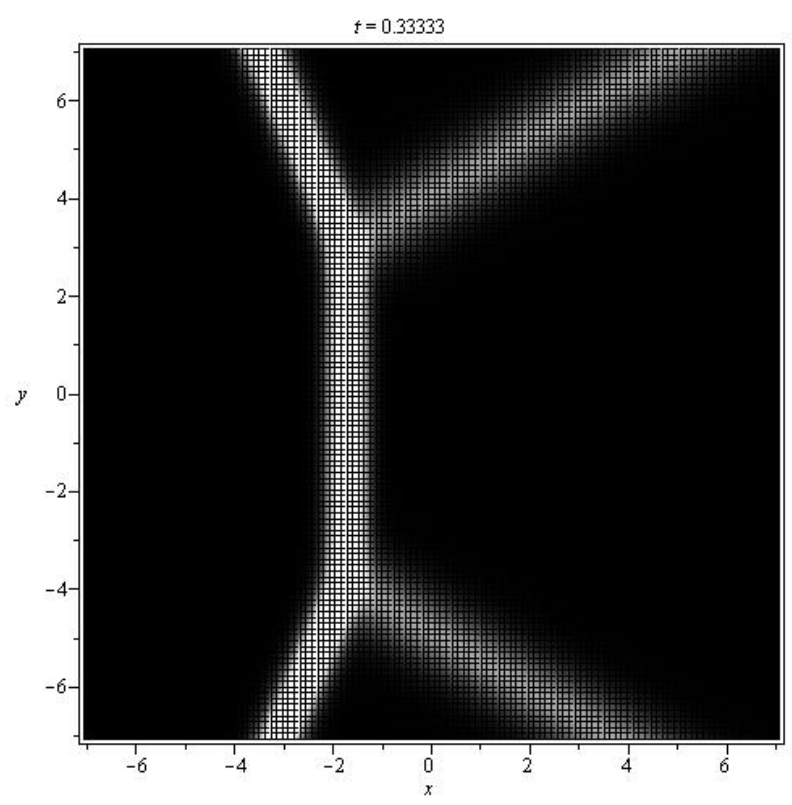

Figure 5. The length of the stem wave is increasing.

Using (2.3), one has

$$
\begin{gathered}
\sin \alpha_{3}=\sin \left(\frac{\alpha_{3}+\alpha_{4}}{2}-\frac{\alpha_{4}-\alpha_{3}}{2}\right)=\frac{(1-\kappa) \tan \varphi_{C}}{\sqrt{\left[1+\left(\tan \varphi_{C}\right)^{2}\right]\left[1+\kappa^{2}\left(\tan \varphi_{C}\right)^{2}\right]}}, \\
\cos \alpha_{4}=\cos \left(\frac{\alpha_{3}+\alpha_{4}}{2}+\frac{\alpha_{4}-\alpha_{3}}{2}\right)=\frac{1-\kappa\left(\tan \varphi_{C}\right)^{2}}{\sqrt{\left[1+\left(\tan \varphi_{C}\right)^{2}\right]\left[1+\kappa^{2}\left(\tan \varphi_{C}\right)^{2}\right]}}, \\
\sin \alpha_{3}+\sin \alpha_{4}=2 \sin \frac{\alpha_{3}+\alpha_{4}}{2} \cos \frac{\alpha_{3}-\alpha_{4}}{2}=\frac{2 \tan \varphi_{C}}{\sqrt{\left[1+\left(\tan \varphi_{C}\right)^{2}\right]\left[1+\kappa^{2}\left(\tan \varphi_{C}\right)^{2}\right]}}, \\
4 \cos ^{2} \alpha_{4}-1=\frac{4\left[1-\kappa\left(\tan \varphi_{C}\right)^{2}\right]^{2}}{\left[1+\left(\tan \varphi_{C}\right)^{2}\right]\left[1+\kappa^{2}\left(\tan \varphi_{C}\right)^{2}\right]}-1 \\
=\frac{3+\left(\tan \varphi_{C}\right)^{2}\left[3 \kappa^{2}\left(\tan \varphi_{C}\right)^{2}-\kappa^{2}-8 \kappa-1\right]}{\left[1+\left(\tan \varphi_{C}\right)^{2}\right]\left[1+\kappa^{2}\left(\tan \varphi_{C}\right)^{2}\right]} .
\end{gathered}
$$

A simple calculation yields using (2.4)

$$
\begin{aligned}
& y=8 t \epsilon \tan \varphi_{C} \frac{1-\kappa}{\left[1+\left(\tan \varphi_{C}\right)^{2}\right]\left[1+\kappa^{2}\left(\tan \varphi_{C}\right)^{2}\right]}=4 t A_{[1,4]} \frac{1-\kappa}{\left(1+\kappa^{2}\right)\left(\tan \varphi_{C}\right)}, \\
& \tan \chi=\frac{y}{x}=\frac{8(1-\kappa) \tan \varphi_{C}}{3+\left(\tan \varphi_{C}\right)^{2}\left[3 \kappa^{2}\left(\tan \varphi_{C}\right)^{2}-\kappa^{2}-8 \kappa-1\right]} .
\end{aligned}
$$

Hence one knows that the length of $[1,4]$-soliton is linear with time and its end points will lie in a line having slope $\pm \tan \chi$ (see Figs. 4 and 5). Furthermore, from (2.5), one gets that the $[1,4]$-soliton moves to the right if $\alpha_{4}<\frac{\pi}{3}$, and moves to the left if $\alpha_{4}>\frac{\pi}{3}$. In particular, if $\alpha_{4}=\frac{\pi}{3}$ or by $(2.7)$

$$
3+\left(\tan \varphi_{C}\right)^{2}\left[3 \kappa^{2}\left(\tan \varphi_{C}\right)^{2}-\kappa^{2}-8 \kappa-1\right]=0 .
$$

then [1,4]-soliton's length is increasing along the $y$-axis. When $\kappa=1$ ( or $\alpha_{3}=0$ ), one has $A=\frac{\epsilon}{2}$ by $(2.8)$ and $\alpha_{4}=\frac{\pi}{3}$. In this special case, the soliton is fixed. It is different from the KP-(II) case $[18,20]$. 


\section{Relations with $V$-shape initial value waves}

In this section, we investigate some relations with the $V$-shape initial value wave for the NovikovVeselov equation (1.1), $\epsilon$ being fixed, as compared with the KP-(II) case [16, 17, 18, 20]. The main purpose is to study the interactions between line solitons, especially for the meaning of the critical angle $\varphi_{C}$.

Recalling the one-soliton solution (1.10) and (1.11), one considers the initial data given in the shape of $V$ with amplitude $A$ and the oblique angle $\varphi_{I}<0$ (measured in the clockwise sense from the $y$-axis):

$$
A \operatorname{sech}^{2}\left[\sqrt{2 A} \cos \varphi_{I}\left(x-|y| \tan \varphi_{I}\right)\right] .
$$

For simplicity, one considers $A \leq 2 \epsilon$. We notice here the $V$-shape initial wave is in the negative $x$ region. The main idea is that we can think the initial value wave as a part of Mach-type soliton (2.1) or $O$-type soliton [8], that is, $c=0$ in (2.1). In order to identify those soliton solutions from the $V$-shape (3.1), we denote them as $\left[i^{+}, j^{+}\right]$-soliton for $y \gg 0$ and $\left[i_{-}, j_{-}\right]$soliton for $y \ll 0$. Solitons for $y \rightarrow \pm \infty$ have by $(1.10)$

$$
\begin{aligned}
& A=2 \epsilon \sin ^{2} \frac{\alpha_{j^{+}}-\alpha_{i^{+}}}{2}=2 \epsilon \sin ^{2} \frac{\alpha_{i_{-}}-\alpha_{j_{-}}}{2}, \\
& \varphi_{I}=\frac{\alpha_{j^{+}}+\alpha_{i^{+}}}{2}=-\left(\frac{\alpha_{i_{-}}+\alpha_{j_{-}}}{2}\right) .
\end{aligned}
$$

Assume that $i^{+}<j^{+}$and $i_{-}>j_{-}$. Then symmetry gives

$$
\alpha_{i^{+}}=-\alpha_{i_{-}}, \quad \alpha_{j^{+}}=-\alpha_{j_{-}} .
$$

Using the parameter $(2.3)[5,8,26]$

$$
\kappa=\frac{\left|\tan \varphi_{I}\right|}{\sqrt{\frac{A}{2 \epsilon-A}}}=\frac{\left|\tan \varphi_{I}\right|}{\tan \varphi_{C}},
$$

one can yield, noticing that $\varphi_{C}=\frac{\alpha_{j^{+}}-\alpha_{i^{+}}}{2}=\frac{\alpha_{i_{-}}-\alpha_{j_{-}}}{2}=\arctan \sqrt{\frac{A}{2 \epsilon-A}}$ from (3.2),

- $\kappa \geq 1 \Rightarrow\left|\varphi_{I}\right| \geq \varphi_{C} \Rightarrow-\frac{\pi}{2} \leq \alpha_{i^{+}}<\alpha_{j^{+}}<\alpha_{j^{-}}<\alpha_{i^{-}} \leq \frac{\pi}{2}$ (O-type),

- $0<\kappa<1 \Rightarrow\left|\varphi_{I}\right|<\varphi_{C} \Rightarrow-\frac{\pi}{2} \leq \alpha_{i^{+}}<\alpha_{j^{-}}<\alpha_{j^{+}}<\alpha_{i^{-}} \leq \frac{\pi}{2}$ (Mach-type).

We remark here that if $\kappa=1$ (or $\alpha_{3}=0$ ), then it is of $O$-type by (2.4) and (2.6). One can see that if the angle $\varphi_{I}$ is small, then an intermediate wave called the Mach stem ([1,4]-soliton) appears. The Mach stem, the incident wave ([1,3]-soliton) and the reflected wave ([3,4]-soliton) interact resonantly, and those three waves form a resonant triple. It is similar to the KP-(II) case [16].

Let's compute the maximal amplitude of the Mach stem ([1,4]-soliton) for fixed amplitude $A$ and $\epsilon$. By (2.4), a simple calculation shows that

$$
\frac{d A_{[1,4]}}{d \kappa}=A \frac{2(\kappa+1)\left[1-\kappa\left(\tan \varphi_{C}\right)^{2}\right]}{\left[1+\kappa^{2}\left(\tan \varphi_{C}\right)^{2}\right]^{2}} .
$$

Hence one can see that when $\kappa=1 /\left(\tan \varphi_{C}\right)^{2}$, that is,

$$
\left(\tan \varphi_{C}\right)\left(\left|\tan \varphi_{I}\right|\right)=1
$$


the Mach stem has the maximal amplitude. Consequently, if

$$
\varphi_{C}+\varphi_{I}=\frac{\pi}{2}
$$

then one obtains by (3.4), recalling that $0<\kappa<1$ (or $\tan \varphi_{C}>1$, i.e., $A>\epsilon$ ),

$$
A_{[1,4]}^{\max }=A\left(1+\frac{1}{\left(\tan \varphi_{C}\right)^{2}}\right)=2 \epsilon<2 A .
$$

Therefore one sees that from (3.4), $A$ and $\epsilon$ being fixed,

- $0<\kappa<\frac{1}{\left(\tan \varphi_{C}\right)^{2}}$, the amplitude $A_{[1,4]}$ (stem wave) is increasing;

- $\kappa=\frac{1}{\left(\tan \varphi_{C}\right)^{2}}($ or $(3.5))$, the amplitude $A_{[1,4]}$ has the maximal value $2 \epsilon$;

- $\frac{1}{\left(\tan \varphi_{C}\right)^{2}}<\kappa<1$, the amplitude $A_{[1,4]}$ is decreasing.

It is noteworthy that the maximal amplitude is independent of $A$. Also, we know that the maximal amplitude of Mach stem for NV equation is less than twice of the incident wave's one; however, for the KP equation (shallow water waves), the Mach stem's amplitude can be four times of the incident wave's one [18]. This is the different point from the case of the KP equation.

On the other hand, one can see that for $\kappa>1$ ( $O$-type) we have $0 \leq \frac{\alpha_{j^{+}}-\alpha_{i^{+}}}{2} \leq \frac{\pi}{4}$ by (3.3), that is, $A \leq \epsilon$. Thus, if we choose $A$ such that

$$
\epsilon<A \leq 2 \epsilon,
$$

we get $\frac{\pi}{2}<\alpha_{i^{-}} \leq \pi$; therefore, under the condition (3.7), the initial value wave (3.1) would develop into a singular $O$-type soliton by $(2.1)(c=0)$ when $\epsilon$ is fixed. On the other hand, when $\left|\varphi_{I}\right| \leq \frac{\pi}{2}, A$ and $\kappa$ are fixed, one can choose

$$
\epsilon=\frac{A}{2}\left[1+\left(\frac{\kappa}{\tan \varphi_{I}}\right)^{2}\right] \geq \frac{A}{2} .
$$

Then we can obtain regular soliton solutions.

Finally, from (2.5) one remarks that the [1,4]-soliton (stem wave) moves to the right if $\alpha_{4}<\frac{\pi}{3}$, and moves to the left if $\alpha_{4}>\frac{\pi}{3}$. The former case is different from the KP equation (shallow water waves); i.e., the stem wave moves with the same side of incident wave for the KP equation. On the other hand, if we replace the condition (2.2) by $A=A_{[3,4]}=A_{[2,1]} \leq 2 \epsilon$, then by (1.11) the $[3,4]$-soliton (the incident wave) has smaller amplitude than the [1,3]-soliton's one (the reflected wave). But this is not physically interesting.

\section{Concluding remarks}

One investigates the Mach-type (or (3142)-type) soliton of the Novikov-Veselov equation. The Mach stem ([1,4]-soliton), the incident wave ([1,3]-soliton) and the reflected wave ([3,4]-soliton) form a resonant triple. From (3.6), we see that the amplitude of Mach stem is less than two times of the one of the incident wave, which is different from the KP equation [18]; moreover, the length of the Mach stem is computed and show it is linear with time (2.6). On the other hand, one uses the parameter $\kappa(2.3)$ to describe the critical behavior for the $O$-type and Mach-type solitons and notices that it depends on the the fixed parameter $\epsilon$. We see that the amplitude $A$ of the incident wave is small than $2 \epsilon$; furthermore, if $\epsilon<A<2 \epsilon$, then the soliton will be singular. Now, a natural question is: what happens if $A>2 \epsilon$ when $\epsilon$ is fixed in (1.1)? Another 
question is the minimal completion [20]. It means the resulting chord diagram has the smallest total length of the chords. This minimal completion can help us study the asymptotic solutions and estimate the maximum amplitude generated by the interaction of those initial waves. A numerical investigation of these issues will be published elsewhere.

\section{Acknowledgements}

The author thanks the referees for their valuable suggestions. This work is supported in part by the National Science Council of Taiwan under Grant No. NSC 102-2115-M-606-001.

\section{References}

[1] Ablowitz M.J., Baldwin D.E., Nonlinear shallow ocean-wave soliton interactions on flat beaches, Phys. Rev. E 86 (2012), 036305, 5 pages, arXiv:1208.2904.

[2] Athorne C., Nimmo J.J.C., On the Moutard transformation for integrable partial differential equations, Inverse Problems 7 (1991), 809-826.

[3] Biondini G., Chakravarty S., Soliton solutions of the Kadomtsev-Petviashvili II equation, J. Math. Phys. 47 (2006), 033514, 26 pages, nlin.SI/0511068.

[4] Bogdanov L.V., Veselov-Novikov equation as a natural two-dimensional generalization of the Korteweg-de Vries equation, Theoret. and Math. Phys. 70 (1987), 219-223.

[5] Chakravarty S., Kodama Y., Soliton solutions of the KP equation and application to shallow water waves, Stud. Appl. Math. 123 (2009), 83-151, arXiv:0902.4423.

[6] Chakravarty S., Lewkow T., Maruno K.I., On the construction of the KP line-solitons and their interactions, Appl. Anal. 89 (2010), 529-545, arXiv:0911.2290.

[7] Chang J.H., On the $N$-solitons solutions in the Novikov-Veselov equation, SIGMA 9 (2013), 006, 13 pages, arXiv:1206.3751.

[8] Chang J.H., The interactions of solitons in the Novikov-Veselov equation, arXiv:1310.4027.

[9] Dubrovsky V.G., Topovsky A.V., Basalaev M.Y., New exact multi line soliton and periodic solutions with constant asymptotic values at infinity of the NVN integrable nonlinear evolution equation via dibar-dressing method, arXiv:0912.2155.

[10] Grinevich P.G., The scattering transform for the two-dimensional Schrödinger operator with a potential that decreases at infinity at fixed nonzero energy, Russ. Math. Surv. 55 (2000), 1015-1083.

[11] Grinevich P.G., Manakov S.V., Inverse problem of scattering theory for the two-dimensional Schrödinger operator, the $\bar{\partial}$-method and nonlinear equations, Funct. Anal. Appl. 20 (1986), 94-103.

[12] Hu H.-C., Lou S.-Y., Construction of the Darboux transformaiton and solutions to the modified NizhnikNovikov-Veselov equation, Chinese Phys. Lett. 21 (2004), 2073-2076.

[13] Hu H.-C., Lou S.-Y., Liu Q.-P., Darboux transformation and variable separation approach: the NizhnikNovikov-Veselov equation, Chinese Phys. Lett. 20 (2003), 1413-1415, nlin.SI/0210012.

[14] Ishikawa M., Wakayama M., Applications of minor-summation formula. II. Pfaffians and Schur polynomials, J. Combin. Theory Ser. A $8 \mathbf{8}$ (1999), 136-157.

[15] Kazeykina A.V., Novikov R.G., Large time asymptotics for the Grinevich-Zakharov potentials, Bull. Sci. Math. 135 (2011), 374-382, arXiv:1011.4038.

[16] Kodama Y., KP solitons in shallow water, J. Phys. A: Math. Theor. 43 (2010), 434004, 54 pages, arXiv:1004.4607.

[17] Kodama Y., KP solitons and Mach reflection in shallow water, arXiv:1210.0281.

[18] Kodama Y., Lectures delivered at the NSF/CBMS Regional Conference in the Mathematical Sciences "Solitons in Two-Dimensional Water Waves and Applications to Tsunami" (UTPA, May 20-24, 2013), available at http://faculty.utpa.edu/kmaruno/nsfcbms-tsunami.html.

[19] Kodama Y., Maruno K.-I., N-soliton solutions to the DKP equation and Weyl group actions, J. Phys. A: Math. Gen. 39 (2006), 4063-4086, nlin.SI/0602031.

[20] Kodama Y., Oikawa M., Tsuji H., Soliton solutions of the KP equation with $V$-shape initial waves, J. Phys. A: Math. Theor. 42 (2009), 312001, 9 pages, arXiv:0904.2620. 
[21] Kodama Y., Williams L., KP solitons, total positivity, and cluster algebras, Proc. Natl. Acad. Sci. USA 108 (2011), 8984-8989, arXiv:1105.4170.

[22] Kodama Y., Williams L., The Deodhar decomposition of the Grassmannian and the regularity of KP solitons, Adv. Math. 244 (2013), 979-1032, arXiv:1204.6446.

[23] Kodama Y., Williams L., KP solitons and total positivity for the Grassmannian, Invent. Math. 198 (2014), 637-699, arXiv:1106.0023.

[24] Manakov S.V., The method of the inverse scattering problem, and two-dimensional evolution equations, Russian Math. Surveys 31 (1976), no. 5, 245-246.

[25] Matveev V.B., Salle M.A., Darboux transformations and solitons, Springer Series in Nonlinear Dynamics, Springer-Verlag, Berlin, 1991.

[26] Miles J.W., Resonantly interacting solitary waves, J. Fluid Mech. 79 (1977), 171-179.

[27] Nimmo J.J.C., Darboux transformations in $(2+1)$-dimensions, in Applications of Analytic and Geometric Methods to Nonlinear Differential Equations (Exeter, 1992), NATO Adv. Sci. Inst. Ser. C Math. Phys. Sci., Vol. 413, Kluwer Acad. Publ., Dordrecht, 1993, 183-192.

[28] Novikov S.P., Veselov A.P., Two-dimensional Schrödinger operator: inverse scattering transform and evolutional equations, Phys. D 18 (1986), 267-273.

[29] Ohta Y., Pfaffian solutions for the Veselov-Novikov equation, J. Phys. Soc. Japan 61 (1992), 3928-3933.

[30] Veselov A.P., Novikov S.P., Finite-gap two-dimensional potential Schrödinger operators. Explicit formulas and evolution equations, Sov. Math. Dokl. 30 (1984), 588-591.

[31] Yeh H., Li W., Kodama Y., Mach reflection and KP solitons in shallow water, Eur. Phys. J. ST 185 (2010), 97-111, arXiv:1004.0370. 